\title{
Comparative Study of Single and Double Ionization of Helium by Ion Impact
}

\author{
D. Fischer ${ }^{1}$, M. Schulz ${ }^{2}$, R. Moshammer ${ }^{1}$, and J. Ullrich ${ }^{1}$ \\ ${ }^{1}$ Max-Planck Institut für Kernphysik, Saupfercheckweg 1, D-69117 Heidelberg, Germany \\ ${ }^{2}$ University of Missouri-Rolla, Physics Department and Laboratory for Atomic, Molecular, \\ and Optical Research, Rolla, Missouri 65409, USA
}

We present three-dimensional angular distributions of the sum momentum vector of electrons emitted in double ionization in $6 \mathrm{MeV} p+\mathrm{He}$ collisions for fully determined kinematics. The results are directly compared to corresponding data for single ionization. Qualitative similarities between single and double ionization are observed. However, we demonstrate that based on these similarities it cannot be concluded that the collision dynamics underlying both processes are similar as well. Furthermore, we find that the projectile - target nucleus interaction, which we found to be surprisingly important for single ionization earlier, is even more significant for double ionization. 


\section{Introduction}

Over the last decade, a variety of new developments in studies of single and multiple ionization by charged particle impact was reported. Theoretically, rapid progress has been achieved in calculating fully differential single ionization cross sections, especially for electron impact, using a variety of approaches like convergent close coupling (CCC) [1] or exterior complex scaling (ECS) [2]. For ion impact refined continuum distorted wave eikonal initial state models (CDW-EIS) had some success [3,4]. For double ionization calculated fully differential cross sections (FDCS) became available only very recently [57]. On the experimental side, the development of cold target recoil-ion momentum spectroscopy (COLTRIMS) initiated a new generation of kinematically complete experiments on ionization processes [8,9].

In the case of single ionization by electron impact very good agreement between experiment and theory has now been achieved for electrons emitted into the scattering plane, spanned by the initial and final projectile momentum vectors, even very close to the threshold [10]. However, for ion impact at very large perturbations $\eta=Q_{p} / v_{p}$ (projectile charge to velocity ratio), which are not accessible for electron impact, large discrepancies were found between experiment and theory $[11,12]$. More seriously, even for a very small perturbation, where the theoretical description should be much easier than at large perturbation, significant discrepancies were observed for electrons emitted out of the scattering plane $[13,14]$. Although these results showed that single ionization is not as well understood as was hoped previously, most of the features observed in the FDCS are either reproduced by theory or can be at least related to some specific ionization mechanism. 
For double ionization, in contrast, we are just at the beginning of understanding the basic features observed in the experiments. Fully differential cross section measurements are still rare, both for electron [15-17] and ion impact [18]. As a result, much of the information that is currently available comes from cross sections integrated over various parameters [e.g. 19-21] or even from total cross sections [22]. The theoretical difficulties in describing double ionization are rooted in the necessity to treat electron-electron correlation effects simultaneously with the dynamics of the two-center potential generated by the projectile and the target nucleus. Schulz et al. $[23,24]$ developed a new method of analyzing electron-electron correlations separately from the collision dynamics. Likewise, it was shown that certain quantities, like the projectile energy loss [25] or the electron sum momentum distribution [26], can to some extent be described independently of electronelectron correlation effects. The four-body problem (2 electrons and 2 nuclei) is thereby effectively reduced to a three-body problem treating the two electrons as one quasi-particle.

Obviously, the FDCS contain the most detailed information about the collision. Furthermore, the basic features in these cross sections for single ionization are amazingly simple and can to a large extent be interpreted within a first order model, at least at small perturbation [27]. For double ionization, on the other hand, the structures in the FDCS are much more complex. Here, even for small perturbations it is not clear that a first order treatment is adequate. For example, data obtained for $2 \mathrm{keV} \mathrm{e}+\mathrm{He}$ (corresponding to a perturbation of $\eta=0.08$ ) did not reflect the symmetry properties with respect to the momentum transfer $\mathrm{q}$ (defined as the difference between the initial and final projectile momenta) which necessarily must be satisfied by any first-order calculation [16]. This 
makes even a qualitative interpretation of the FDCS significantly more complicated than in the case of single ionization.

Given these difficulties in interpreting the FDCS for double ionization and the success in describing certain quantities by treating both electrons as one quasi-particle led us to attempt the following approach to analyze double ionization data: we determined the sum momentum vector $\mathrm{P}=\mathrm{p}_{1}+\mathrm{p}_{2}$ of both electrons thereby again treating the collision as an effective three-body system. We then analyzed the FDCS for the ejection of the quasiparticle composed of the two electrons. This method was employed recently for double ionization of Magnesium by electron impact for the case that $\mathrm{P}$ lies in the scattering plane [28]. Here, we present a generalization of this approach in that we analyzed complete three-dimensional images of the angular distribution of P. This enables us to directly compare the collision dynamics in double ionization to corresponding data for single ionization. Furthermore, we can test to what extent electron-electron correlations can be separated from the collision dynamics. However, it is not possible to obtain detailed information about such correlations.

\section{Experiment}

The experiment was performed at the Max-Planck-Institut für Kernphysik in Heidelberg. A bunched $6 \mathrm{MeV}$ p beam (pulse length less than $1 \mathrm{~ns}$ ) was collimated to a size of about $1 \times 1$ $\mathrm{mm}^{2}$ and crossed with a He beam from a supersonic gas jet. The very cold He beam was obtained from a three stage supersonic gas jet. At the intersection point with the projectile beam the gas jet had a diameter of about $1 \mathrm{~mm}$ and a thickness of $10^{11} / \mathrm{cm}^{2}$. 
The electrons and recoil ions were momentum-analyzed by the same spectrometer system. It consists of two parallel resistive plates $22 \mathrm{~cm}$ in length and separated by a distance of $7 \mathrm{~cm}$ which are oriented along the projectile beam axis. An electric field was generated by applying a voltage of $60 \mathrm{~V}$ across the plates so that the electrons were extracted parallel and the recoil ions anti-parallel to the projectile beam direction. After traversing a $22 \mathrm{~cm}$ long field free drift tube following the extraction region, the recoil ions and electrons were detected by two two-dimensional position-sensitive channel plate detectors with diameters of 40 and $80 \mathrm{~mm}$, respectively.

The extraction field was not strong enough to guide a sufficiently large fraction of the electrons onto the detector. Therefore, a uniform magnetic field of $14 \mathrm{G}$ in the same direction as the electric extraction field was generated by two Helmholtz coils. As a result, the electrons were forced into cyclotron motion with a radius proportional to the transverse momentum component of the electrons. For transverse momenta of less than 2.0 a.u. the cyclotron radius was small enough for the electrons to hit the detector.

In order to simultaneously record the time of flight and position information of both electrons emitted in the same collision with a single detector, a delay line anode in conjunction with a multi-hit time to digital converter (TDC) was used [29]. The multi-hit resolution, i.e. the minimum time delay between two electrons required to identify them as separate particles, was $10 \mathrm{~ns}$. As a result of this deadtime, a small fraction (corresponding to small momentum differences between the two electrons) of the 6-dimensional momentum space was not recorded.

The electron detector was set in coincidence with the recoil ion detector. A timing signal from the projectile beam buncher was used as a reference for the coincidence time 
measurement. From the coincidence the time of flight of the recoil ions and the ionized electrons from the collision to the respective detector were obtained with a resolution of better than $1 \mathrm{nsec}$. From the time of flight, in turn, the momentum component in the longitudinal direction was determined. The two transverse components were deduced from the position information. The momentum resolution for the recoil ions was 0.1 a.u. in the longitudinal direction and 0.3 a.u. in the transverse direction; the corresponding values for the electrons are 0.01 and 0.1 a.u. In the transverse direction the electron momentum resolution depends on both the longitudinal and transverse components and the value provided above represents an approximated average.

\section{Results and Discussion}

The FDCS for single ionization depend on 5 and those for double ionization even on 8 independent parameters. For such a highly differential quantity it is not easy to find an informative form of presentation. One very common presentation is to fix the magnitude of the momentum transfer and the energy of the electron(s) and to plot the FDCS for electrons emitted into the scattering plane as a function of the polar emission angle (measured relative to the initial beam direction) of the electron(s) [27]. Recently, we reported threedimensional plots of such fully differential angular electron distributions for single ionization [13], which enables one to present the data for all electron emission planes (spanned by $\mathrm{p}_{\mathrm{o}}$ and the electron momentum $\mathrm{p}_{\mathrm{e}}$ ) simultaneously. Here, we chose an analogue three-dimensional presentation for double ionization. However, instead of generating such a plot for individual electrons, we present the three-dimensional angular distribution for the sum momentum vector of both electrons P. More specifically, we plot 
the FDCS as a function of the polar and azimuthal angles $\Theta$ and $\varphi$ of $\mathrm{P}$ in spherical coordinates, where $\varphi$ denotes the angle between the scattering plane and the electron emission plane. This is shown in Fig. 1 for sum electron energy and momentum transfer combinations of $6.5 \mathrm{eV} / 0.75$ a.u., $11 \mathrm{eV} / 1.1$ a.u., and $15 \mathrm{eV} / 1.5$ a.u. For comparison, we show in the bottom of Fig. 1 the corresponding three-dimensional plots for single ionization for the same collision system and same kinematic conditions. The arrows labeled $p_{o}$ and $q$ indicate the direction of the initial projectile beam and of the momentum transfer, respectively.

The features observed for single ionization are essentially the same as those we reported earlier for $100 \mathrm{MeV} / \mathrm{amu} \mathrm{C}^{6+}+\mathrm{He}$ collisions [13], i.e. a pronounced maximum in the direction of $\mathrm{q}$ (known as the binary peak) and a structure with a slight maximum in the direction of $-\mathrm{q}$ (known as the recoil peak). However, the sharp minimum that separates the recoil peak from the binary peak in calculated FDCS [13] is strongly filled up in the experimental data. As a result, the shape of the recoil peak resembles more the one of a ring around $\mathrm{p}_{\mathrm{o}}$ rather than the one of a peak. The binary peak can be explained in terms of a binary interaction with the projectile. The recoil peak, has been interpreted as a double scattering process [30]: the electron, initially emitted approximately in the direction of $\mathbf{q}$, on its path out of the atom elastically backscatters from the recoil ion, which picks up most of the momentum transferred from the projectile. However, it has been pointed out that a second-order process involving the projectile - target nucleus interaction may also contribute to the recoil peak [13]. 
For large q, the structures we find for double ionization are quite similar to those in single ionization. Again, the main feature is a pronounced binary peak in the direction of $\mathrm{q}$ which is accompanied by a ring-like shaped recoil peak in the direction of -q. However, the recoil to binary peak intensity ratio is larger than for single ionization. Furthermore, these structures are significantly broader than for single ionization and the width increases with decreasing q. At $\mathrm{q}=0.75$ a.u. this broadening is already so pronounced that the peak structures are just barely discernable and the data for single and double ionization no longer show the similarities that we observe for large q. This is qualitatively the same behavior that was reported by El-Marji et al. [28] for double ionization of $\mathrm{Mg}$ by electron impact.

For the sum electron momentum in double ionization, the binary peak can be interpreted in the same way as for single ionization. However, here either a binary interaction may occur between the projectile and only one electron or the projectile interacts with both electrons independently. The former case requires an electron-electron interaction to ionize the second electron. It should be noted that this interaction cannot alter the direction of $\mathrm{P}$ since it represents an internal force to the two-electron subsystem. Likewise, the recoil peak may be explainable by a similar backscattering model as applied to the case of single ionization. However, for the sum electron momentum in double ionization to point in the direction of $-\mathrm{q}$ either the faster or both of the ionized electrons would have to be backscattered by the target nucleus. A less complex, and therefore perhaps a more likely mechanism is the process involving the projectile - target nucleus interaction which was mentioned above. Here, double ionization starts off the same way as the process leading to the binary peak, however, then the projectile elastically scatters of the target nucleus leading to a rotation of $\mathrm{q}$ by any angle between 0 and $180^{\circ}$ about the 
initial projectile beam axis (it should be noted that even a rotation of $\mathrm{q}$ by $180^{\circ}$ corresponds to a very small scattering angle of the projectile).

At first glance, the similarities between single and double ionization at large q seem to support the conclusion of Perumal et al. [26] that at least certain aspects of double ionization can be described within an effective three-particle problem treating the electrons as a single quasi-particle. However, before making conclusions about the collision dynamics based on these similarities, it is important to carefully analyze which potential differences in the collision dynamics for single and double ionization would even be visible in this comparison. We start by noting that it is common to distinguish between correlated and uncorrelated double ionization mechanisms [31]. In a correlated process, the projectile only needs to interact with one electron directly and the second electron is ionized by an electron - electron interaction. In an uncorrelated process, in contrast, the projectile interacts with both electrons independently. In a qualitative description, the projectile target nucleus interaction is, rightfully or not, usually neglected in both processes. Initially, we therefore investigate the question whether the two processes can lead to different features in the angular distribution of $\mathrm{P}$ under the assumption that the recoil - ion momentum is small. Furthermore, we neglect the initial momentum distribution of the electrons in the He ground state. Using momentum conservation it is straight forward to show that then $\mathrm{P}=\mathrm{q}$ for both processes.

The analysis above shows that studying three-dimensional angular distributions of $\mathrm{P}$ is not a sensitive method to distinguish between correlated and uncorrelated double ionization. Furthermore, it demonstrates that any deviation in the direction of $\mathrm{P}$ from $\mathrm{q}$ must be compensated by a non-zero recoil-ion momentum. Apart from the initial 
momentum distribution in the ground state of the He atom, the recoil ion can only acquire momentum through an interaction with the projectile or through the backscattering process of the electrons leading to the recoil peak. Therefore, our data may be very suitable to compare the effects of the projectile - target nucleus interaction on the collision dynamics for single and double ionization.

Using a similar analysis as the one described above, El-Marji et al. have argued that the similarities between single and double ionization shows that for large $\mathrm{q}$ the role of the projectile - target nucleus interaction is insignificant and that it implies a similar mechanism for both processes [28]. Here, we point out that such a conclusion should be treated very cautiously. First of all, as shown above, these similarities do not allow for any conclusion at all about the significance of electron-electron correlations, the most important factor which potentially could lead to differences between the single and double ionization mechanisms. Second, while a deviation from a binary peak structure in the direction of $\mathrm{q}$ is a strong indication for significant projectile - target nucleus scattering, the reverse conclusion is not as straight forward. For the case of single ionization, we have recently shown that a strong projectile - target nucleus interaction leads to a ridge in the doubly differential cross sections (DDCS) as a function of the transverse recoil - ion momentum $\mathrm{p}_{\mathrm{rt}}$ and of the transverse momentum transfer $\mathrm{q}_{\mathrm{t}}[14]$. In Fig. 2 we present the corresponding cross sections for double ionization. The plots in the center and on the right show these data for the additional condition that $\mathrm{P}$ lies in the scattering plane (yz-plane) and the perpendicular plane (xz-plane), respectively, and the plot on the left those without any condition on $\mathrm{P}$. For the case that $\mathrm{P}$ lies in the perpendicular plane the triangle below the diagonal in Fig. 2 is kinematically forbidden [14]. Again, a ridge of strongly enhanced 
intensity is observed along the diagonal for which $\mathrm{p}_{\mathrm{rt}}=\mathrm{q}_{\mathrm{t}}$. This ridge extends well into the region (large q) where we observe a clear binary peak in the direction of q. Furthermore, it should be noted that the ridge is already rather prominent in the DDCS without any condition on $\mathrm{P}$, although not quite as pronounced as for $\mathrm{P}$ lying in the perpendicular plane. In fact even the plot for $\mathrm{P}$ lying in the scattering plane, where in single ionization the ridge is essentially absent, shows a clear accumulation of the data around the diagonal. Therefore, we conclude that the existence of a pronounced binary peak is no guarantee that the projectile - target nucleus interaction is insignificant and that this interaction is even more important than in single ionization.

For the case of single ionization of $\mathrm{He}$ by $100 \mathrm{MeV} / \mathrm{amu} \mathrm{C}^{6+}$ impact it was shown that this strong projectile - target nucleus interaction resulted in a peak structure in the FDCS for electrons emitted into the plane perpendicular to $\mathrm{q}$, while the first Born approximation yields an isotropic angular dependence in that plane [13,14]. Given the strong effects due to this interaction in the DDCS of Fig. 2, one might expect to find a similar peak structure in the FDCS for double ionization for the case that the electron sum momentum P lies in the perpendicular plane. Instead, these cross sections, which are shown in Fig. 3, show a similarly isotropic angular dependence as predicted by the first Born approximation. This can probably be explained by the significantly broader initial two-electron momentum distribution of the electrons in the initial ground state of He compared to the one-electron momentum distribution. Indeed, the binary peak and recoil peak in the scattering plane for double ionization are much broader than for single ionization. Since the peak for single ionization in the perpendicular plane is much less pronounced than the binary peak in the scattering plane, it is not surprising that for double ionization the much broader two- 
electron momentum distribution completely washes out the structure in the perpendicular plane.

If the above arguments for the absence of a peak in the perpendicular plane for double ionization are correct, then the FDCS should be nearly cylindrically symmetric about $\mathrm{q}$ because of the symmetry of the momentum distribution of the He ground state. If the initial momentum distribution is the dominant contributor to the width of the binary peak, then even higher order contributions to the ionization process would not significantly break this symmetry about q. This would imply that the azimuthal angular dependence (i.e. the angular dependence in the xy-plane in the coordinate system of Fig. 1) for a fixed polar angle of 0 relative to $\mathrm{q}$ and the polar angular dependence (i.e. the angular dependence in the yz-plane) for a fixed azimuthal angle of 0 relative to q of the FDCS should be the same. Note that for the sake of an easier comparison in shape between these two data sets, here we changed the definition of $\Theta_{\mathrm{e}}=0$ compared to Figs. 1 and 3, where that angle corresponds to the initial projectile beam direction instead of $\mathrm{q}$. These dependencies are shown in Fig. 4. Indeed, both angular dependencies are essentially identical illustrating nearly cylindrical symmetry about q. In contrast, for single ionization we found earlier that the azimuthal angular dependence is significantly broader than the polar angular dependence, i.e. that this symmetry is clearly broken [32]. For the case of single ionization this broken symmetry is evidence for higher-order contributions to the cross sections. However, the reverse conclusion for double ionization is not possible: the approximate cylindrical symmetry about q does not mean that higher-order contributions are negligible 
since we demonstrated the importance of multiple scattering events involving the projectile - target nucleus interaction.

\section{Conclusions}

We have presented three-dimensional angular distributions of the sum momentum of the electrons emitted in double ionization for completely determined kinematics. The data were directly compared to corresponding three-dimensional images for single ionization. Qualitatively, striking similarities were found in that in both cases the main feature is a binary peak pointing in the direction of $\mathrm{q}$ and a ring-like shaped recoil structure. However, in contrast to single ionization, the cross sections are cylindrically symmetric about $\mathrm{q}$ for double ionization, which can probably be attributed to the much broader initial momentum distribution of the electron sum momentum in He (relevant to double ionization) compared to the one-electron momentum distribution (relevant in single ionization).

We demonstrated that conclusions based on these similarities should be drawn cautiously: it cannot be concluded that the dynamics in both process is similar because the angular distribution of the electron sum momentum for double ionization contains very limited information about electron-electron correlations. Furthermore, from the presence of a pronounced binary peak it cannot be concluded that the interaction between the projectile and the target nucleus is unimportant because we showed that this interaction is actually important even in cases where the binary peak is clearly observable. In fact, we conclude that the projectile - target nucleus interaction in double ionization is even more important than in single ionization. Finally, a cylindrical symmetry about q does not necessarily reflect a dominance of first-order contributions to the cross sections. 


\section{Acknowledgements}

One of us (M.S.) is grateful for the hospitality of the Max-Planck-Institut für Kernphysik in Heidelberg. This work was supported by the Leibniz-Programm of the Deutsche Forschungsgemeinschaft and the National Science Foundation under grants PHY-0097902 and INT-0224943. 


\section{Figure Captions}

Fig.1: Three-dimensional angular distributions of the electron sum-momentum vector in double (top) and single ionization (bottom) for $6 \mathrm{MeV} \mathrm{p}+\mathrm{He}$ collisions. The electron sum energy - momentum transfer combinations are (from left to right) $6.5 \mathrm{eV} / 0.75$ a.u., 11 eV/1.1 a.u., and $15 \mathrm{eV} / 1.5$ a.u.

Fig. 2: DDCS (on a logarithmic scale) as a function of the transverse recoil-ion momentum and the transverse momentum transfer for double ionization in $6 \mathrm{MeV} \mathrm{p}+\mathrm{He}$ collisions. The data are shown for the condition that the electron sum-momentum vector lies in the scattering plane (center) and in the plane perpendicular to q (right) and without condition (left).

Fig. 3: Polar angular distribution of the electron sum-momentum vector P (measured relative to the initial beam direction) for the condition that $\mathrm{P}$ lies in the plane perpendicular to q. The electron sum energy and momentum transfers are the same as in Fig. 1.

Fig. 4: Azimuthal angular dependence in the xy-plane of Fig. 1 (open symbols) and the polar angular dependence in the scattering or yz-plane (closed symbols) of the electron sum-momentum vector. Both angles are measured relative to the direction of $\mathrm{q}$. 
[1] Bray I. and Stelbovic A.T., Phys. Rev. Lett. 69, 53 (1992)

[2] Rescigno T.N., Baertschy M., Isaacs W.A., and McCurdy C.W., Science 286, 2474 (1999)

[3] Madison D.H., D. Fischer, M. Foster, M. Schulz, R. Moshammer, S. Jones, and J. Ullrich, Phys. Rev. Lett. 91, 253201 (2003)

[4] Rodriguez V.D. and Barrachina R.O., Phys. Rev. A57, 215 (1998)

[5] Kheifets A., Bray I., Lahmam-Bennani A., Duguet A., and Taouil I., J. Phys. B32, 5047 (1999)

[6] Jones S. and Madison D.H., Phys. Rev. Lett. 91, 073201 (2003)

[7] Berakdar J., Lahmam-Bennani A., and Dal Capello C., Physics Reports 374, 91 (2003)

[8] Ullrich J., Moshammer R., Dorn A., Dörner R., Schmidt L., Schmidt-Böcking H., Rep. Prog. Phys. 66, 1463 (2003)

[9] Dörner R., et al. Physics Reports 330, 95 (2000)

[10] J. Röder, M. Baertschy, and I. Bray, Phys. Rev. A67, 010702(R) (2003)

[11] Schulz M., Moshammer R., Perumal A.N., and Ullrich J., J. Phys. B35, L161 (2002)

[12] Fischer D., Moshammer R., Schulz M., Voitkiv A., and Ullrich J., J. Phys. B36, 3555 (2003)

[13] Schulz M., Moshammer R., Fischer D., Kollmus H., Madison D.H., Jones S., and Ullrich J., Nature 422, 48 (2003)

[14] Schulz M., MoshammerR., Fischer D., and Ullrich J., J. Phys. B36, L311(2003)

[15] Taouil I., Lahmam-Bennani A., Duguet A., and Avaldi L., Phys. Rev. Lett. 81, 4600 (1998)

[16] Dorn A., Kheifets A., Schröter C.D., Najjari B., Höhr C., Moshammer R., and 
J. Ullrich, Phys. Rev. Lett. 86, 3755 (2001)

[17] Dorn A., Kheifets A., Schröter C.D., Najjari B., Höhr C., Moshammer R., and J. Ullrich, Phys. Rev. A65, 032709 (2002)

[18] Fischer D., Moshammer R., Dorn A., Crespo Lopez-Urrutia J.R., Feuerstein B., Höhr C., Schröter C.D., Hagmann S., Kollmus H., Mann R., Bapat B., and Ullrich J., Phys. Rev. Lett. 90, 243201 (2003)

[19] Giese J.P. and Horsdal E., Phys. Rev. Lett. 60, 2018 (1988)

[20] Moshammer R., Ullrich J., Kollmus H., Schmitt W., Unverzagt M., Jagutzki O., Mergel V., Schmidt-Böcking H., Mann R., Woods C.J., and Olson R.E., Phys. Rev. Lett. 77, $1242(1996)$

[21] Bapat B., Keller S., Moshammer R., Mann R., and Ullrich J., J. Phys. B33, 1437 (2000)

[22] Andersen L.H., Hvelplund P., Knudsen H., Møller S.P., Elsener K., Rensfelt K.-G., and Uggerhøj E., Phys. Rev. Lett. 57, 2147 (1986)

[23] Schulz M., Moshammer R., Schmitt W., Kollmus H., Feuerstein B., Mann R., Hagmann S., and Ullrich J., Phys. Rev. Lett. 84, 863 (2000)

[24] Schulz M., Moshammer R., Gerchikov L.G., Sheinerman S.A., and Ullrich J., J. Phys. B34, L795 (2001) L795

[25] Schulz M., Moshammer R., Schmitt W., Kollmus H., Mann R., Hagmann S., Olson R.E., and Ullrich J., J. Phys. B32, L557 (1999)

[26] Perumal A.N., Moshammer R., Schulz M., and Ullrich J., J. Phys. B35, 2133 (2002)

[27] Ehrhardt H., Jung K., Knoth G., and Schlemmer P., Z. Phys. D1, 3 (1986) and references therein 
[28] El-Marji B., Doering J.P., Moore J.H., and Coplan M.A., Phys. Rev. Lett. 83, 1574 (1999)

[29] Moshammer R., Unverzagt M., Schmitt W., Ullrich J., and Schmidt-Böcking H., Nucl. Instrum. Meth. B108, 425 (1996)

[30] Whelan C.T., Allan R.J., Walters H.R.J., and Zhang X., in Whelan C.T., Walters

H.R.J., Lahmam-Bennani A., and Ehrhardt H. (editors), (e,2e) \& related processes, Kluwer, Dordrecht, Series C: Mathematical and Physical Sciences 414, 1 (1993)

[31] McGuire J.H., Phys. Rev. Lett. 49, 1153 (1982)

[32] Madison D.H., Schulz M., Jones S., Foster M., Moshammer R., and Ullrich J., J. Phys. B35, 3297 (2002) 

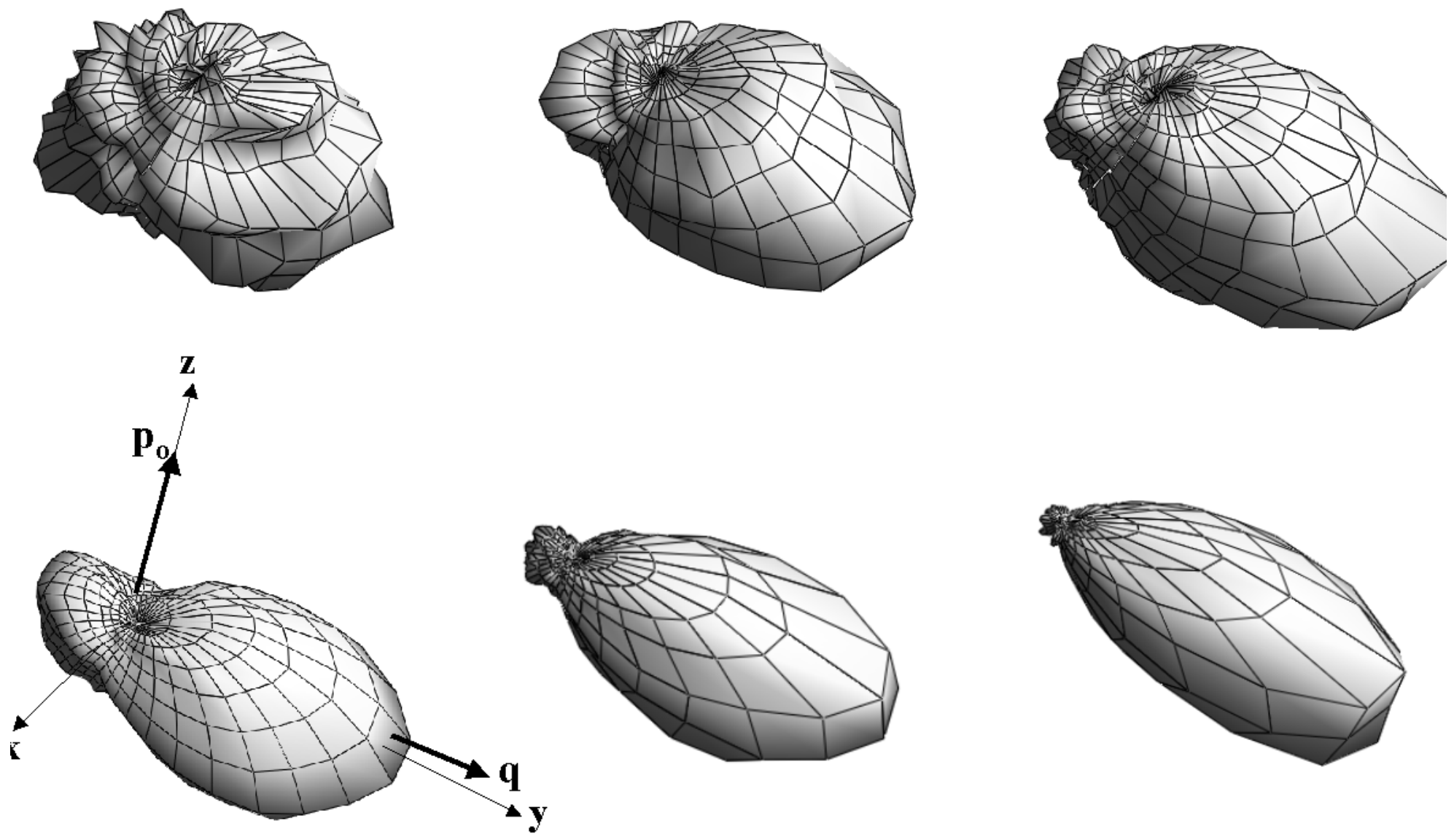

Fig. 1
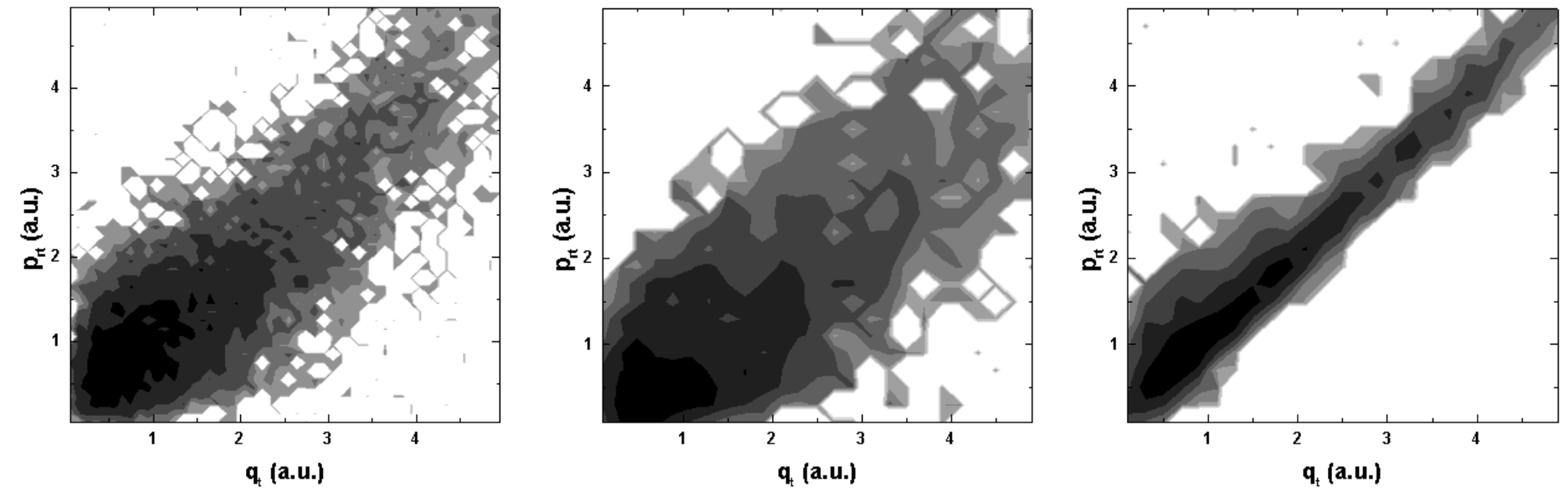

Fig. 2 

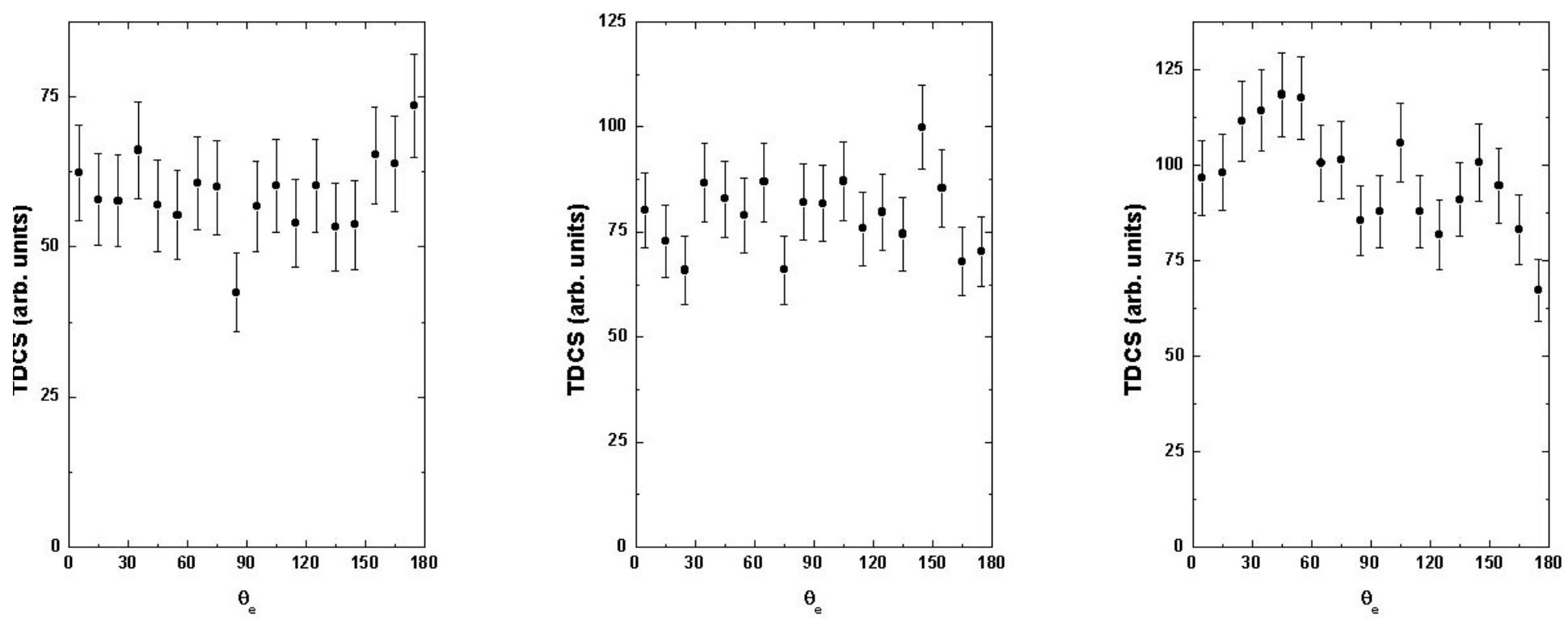

Fig. 3
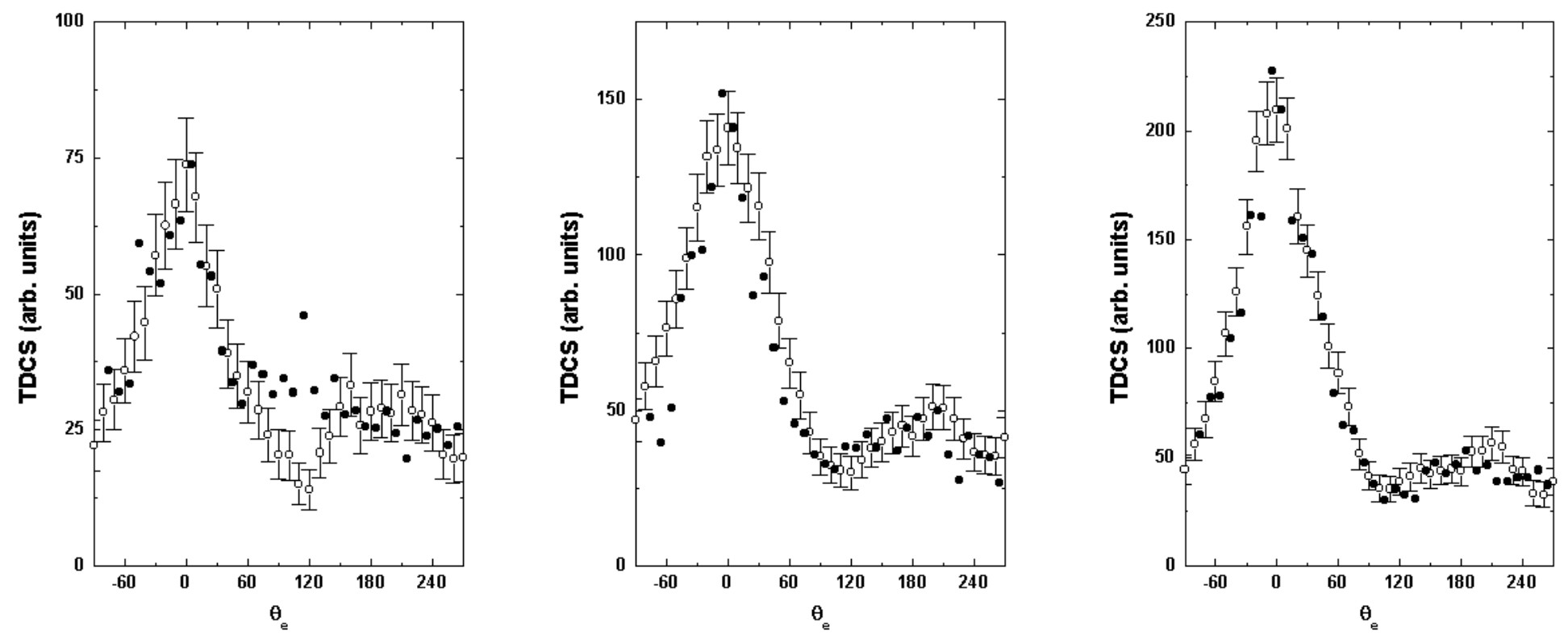

Fig. 4 